\title{
Role of ARHGAP24 in ADP Ribosylation Factor 6 (ARF6)-dependent Pseudopod Formation in Human Breast Carcinoma Cells
}

\author{
SHOTA UEHARA*, KOJI SAITO*, HISAYO ASAMI and YASUTAKA OHTA
}

\author{
Division of Cell Biology, Department of Biosciences, School of Science, Kitasato University, Kanagawa, Japan
}

\begin{abstract}
Background/Aim: The small GTPase ADP ribosylation factor 6 (ARF6) promotes carcinoma cell invasion and metastasis through remodeling of actin cytoskeleton and formation of pseudopod that is regulated by RAC. RHO GTPase activating protein 24 (ARHGAP24), a RAC-specific GTPase activating protein, binds to activated ARF6 and is recruited to the plasma membrane. The aim of the present study was to demonstrate if ARHGAP24 is involved in the ARF6-mediated formation of pseudopods in breast carcinoma cells. Materials and Methods: The formation of pseudopods induced by activated ARF6 was monitored using MDA-MB231 human breast carcinoma cells. The effect of knockdown of endogenous ARHGAP24 by siRNA was examined. Results: Knockdown of ARHGAP24 in MDA-MB-231 carcinoma cells increased the lifespan of pseudopods to retract, which resulted in increased length of pseudopods induced by activated ARF6. ARHGAP24 required a binding site of ARF6 to achieve ARF6dependent actin remodeling. Conclusion: ARHGAP24 may regulate pseudopod formation downstream of activated ARF6 in MDA-MB-231 human breast carcinoma cells.
\end{abstract}

Metastasis is a major clinical problem in cancer and abnormal cell migration and invasion is intimately involved in cancer metastasis $(1,2)$. Cancer cells extend actin-rich surface protrusions (pseudopods) to migrate and actin remodeling is essential for cell shape change and migration (3). RHO family small GTPases control actin cytoskeletal dynamics to induce pseudopod formation in cells. For

\footnotetext{
*These Authors contributed equally to this work.

Correspondence to: Yasutaka Ohta, School of Science, Kitasato University, 1-15-1 Kitasato, Minami-ku, Sagamihara, Kanagawa 252-0373, Japan. Tel: +81 427789401, Fax: +81 427789401, e-mail: yohta@kitasato-u.ac.jp
}

Key Words: Breast cancer, cancer cell migration, small GTPases, actin cytoskeleton. example, RHOA promotes the formation of actin stress fibers and focal adhesions, RAC induces lamellae, and CDC42 stimulates the formation of filopodia (4-6). Previous studies have demonstrated altered expression of RHO GTPases and their regulators in various cancers (7).

The ADP ribosylation factor (ARF6) protein is a small GTPase of the RAS superfamily and is a component of signaling cascades promoting cancer invasion and metastasis (8-11). ARF6 down-regulates E-cadherin expression at cellcell junctions and up-regulates recycling of $\beta 1$ integrin (12, 13). Importantly, ARF6 contributes to cancer cell malignancy through regulation of formation of invadopodia and cancer cell migration $(14,15)$. ARF6 regulates endocytosis and recycling of plasma membrane proteins, and also regulates cortical actin cytoskeleton. Previous studies have demonstrated that ARF6dependent actin remodeling is mediated by RAC (16-20).

ARHGAP24 (also called FilGAP) is a RHO GTPaseactivating protein (GAP) and binds to actin filament crosslinking protein filamin A (21-31). The GAP activity of ARHGAP24 is specific for RAC. ARHGAP24 is phosphorylated by RHO-associated protein kinase (ROCK), an effector of RHOA, and recruited to plasma membranes by binding to activated ARF6 $(25,27)$. ARHGAP24 binds to ARF6 through its pleckstrin-homology $(\mathrm{PH})$ domain and inactivates RAC as a downstream effector of ARF6 (25). We previously showed that ARHGAP24 may regulate carcinoma cell invasion and metastasis by controlling cell shape change during migration (23). Moreover, pathological studies have shown that expression level of ARHGAP24 may affect behavior of human B-cell lymphoma and astrocytoma $(30,31)$.

In this study, we examined the role of ARHGAP24 downstream of activated ARF6 in actin remodeling and cell shape in MDA-MB-231 human breast carcinoma cells.

\section{Materials and Methods}

Cell culture and transfection. MDA-MB-231 Human breast cancer cell line and HEK293 Human embryonic kidney cell line were cultured in Dulbecco's modified Eagle's medium (DMEM) (SigmaAldrich, St. Louis, MO, USA) supplemented with $10 \%$ fetal bovine 
serum (FBS), and 50 units $/ \mathrm{ml}$ penicillin/streptomycin at $37^{\circ} \mathrm{C}$ with $5 \% \mathrm{CO}_{2}$. Cells were transfected with plasmid DNA for $24 \mathrm{~h}$ or ARHGAP24 siRNA for $48 \mathrm{~h}$ using Lipofectamine 2000 (Invitrogen, Carlsbad, CA, USA) according to the manufacturer's instructions. For co-transfection of plasmid DNA and ARHGAP24 siRNA, cells were first transfected with siRNA for $24 \mathrm{~h}$ and then co-transfected with plasmid DNA, followed by additional culture for $24 \mathrm{~h}$. MDAMB-231 cells were cultured on poly-L-lysine-coated coverslips. In some experiments, the cells were cultured on coverslips coated with collagen type I or fibronectin.

Antibodies and regents. Mouse anti-hepatitis A (HA) monoclonal (12CA5), anti- $\alpha$-tubulin monoclonal and rabbit anti-FLAG polyclonal antibodies were purchased from Sigma-Aldrich. Mouse anti-ARF6 monoclonal antibody (3A-1) was purchased from Santa Cruz Biotechnology (Santa Cruz, CA, USA). Rabbit anti-green fluorescent protein (GFP) polyclonal antibody was purchased from MBL (Nagoya, Japan). Rabbit anti-ARHGAP24 polyclonal antibody was prepared as described previously (21). Secondary antibodies conjugated to Alexa Fluor 488 or 568 or Alexa Fluor 568-phalloidin (Invitrogen) and Hoechst 33258 (Dojido Laboratries, Kumamoto, Japan) were also purchased from commercial sources.

The pIRES2-AcGFP (Clonetech, Palo Alto, CA, USA) and pFLAG-CMV-6c (Sigma-Aldrich) plasmids encoding ARF6 Q67L were generated as follows; the ARF6 Q67L coding sequence was polymerase chain reaction (PCR)-amplified using HA-tagged ARF6 Q67L construct (pcDNA3-HA-ARF6 Q67L) (25) as a template and the PCR products were inserted into the pIRES2-AcGFP or pFLAGCMV-6c vector using the EcoRI site and sequenced. MDA-MB-231 cells transfected with ARF6 Q67L-IRES-AcGFP plasmid produce both ARF6 Q67L and GFP proteins and the transfected cells are easily identified using GFP expression as a marker.

The FLAG-tagged ARHGAP24 constructs [wild type (WT), $\triangle \mathrm{PH}, \mathrm{R} 39 \mathrm{C}$, see below] and HA-tagged ARHGAP24 siRNAresistant construct ( $\mathrm{rKD})$ were as described previously $(23,25)$. The ARHGAP24 $\triangle \mathrm{PH}$ (rKD) coding sequence was amplified by PCR and cloned into the pCMV5-HA vector using the EcoRI site. The HA-tagged ARHGAP24 R39C (rKD) construct was generated by introducing point mutations using the QuikChange site-directed mutagenesis kit (Stratagene, La Jolla, CA, USA) as described previously (23). siRNA oligonucleotide duplexes targeting human ARHGAP24 was purchased from Invitrogen. The targeting sequence was: 5'-CAGUGGUAAAUUACAACCUCCUCAA-3' (nt 770-794). This siRNA was found to significantly reduce the expression of endogenous ARHGAP24 in MDA-MB-231 cells (23).

Immunofluorescence and microscopic observation. MDA-MB-231 cells plated on coverslips were washed with phosphate-buffered saline (PBS) and fixed with $3.7 \%$ formaldehyde in PBS at room temperature for $10 \mathrm{~min}$. The fixed cells were then permeabilized with $0.5 \%$ Triton X-100 in PBS for $10 \mathrm{~min}$, then incubated with blocking buffer [10\% Blocking one (Nacarai tesque, Kyoto, Japan) in PBS] for $30 \mathrm{~min}$, and immunostained with primary antibodies in blocking buffer for $1 \mathrm{~h}$ at room temperature. Cells were then washed with PBS and incubated with Alexa Fluor dye-labeled secondary antibodies (Invitrogen) in blocking buffer for $1 \mathrm{~h}$ at room temperature. For visualization of F-actin and nuclei, Alexa Fluor 568-palloidin and Hoechst 33258 were added with secondary antibodies. Cells were washed with PBS and mounted on glass slides then covered with AquaPolyMount (Polysciences, Warrington,
PA, USA) and coverslipped. Cells were observed under an Olympus IX73 fluorescence microscope with $40 \times$ or $60 \times$ objective (Olympus, Tokyo, Japan). Images were acquired by HCImage Live (Hamamatsu photonics, Hamamatsu, Japan) and analyzed by ImageJ (National Institutes of Health, NIH, Bethesda, MD, USA) to measure the length of pseudopods and the elongation factor of cells (the ratio of maximum length to width).

Time-lapse microscopy. MDA-MB-231 cells were seeded on glass bottom dishes and transfected with pIRES2-AcGFP plasmid encoding constitutively activated ARF6 Q67L in the presence or absence of ARHGAP24 siRNA. The cells were examined under Olympus IX81 fluorescence microscope with $10 \times$ objective, and images were acquired at $37^{\circ} \mathrm{C}$ every $10 \mathrm{~min}$ for $12 \mathrm{~h}$ and analyzed by MetaMorph software (Molecular Devices, Sunnyvale, CA, USA) to measure the length and lifespan of pseudopods.

Association of ARHGAP24 and ARF6. Association of ARHGAP24 and ARF6 was determined as described previously (25). Briefly, HEK293 cells were transfected with the HA-ARF6 Q67L and FLAG-ARHGAP24 constructs (WT, $\triangle \mathrm{PH}, \mathrm{R} 39 \mathrm{C}$ ). $24 \mathrm{~h}$ later, the cells were washed twice with PBS, suspended in $500 \mu \mathrm{l}$ of lysis buffer containing $50 \mathrm{mM}$ Tris- $\mathrm{HCl}$ (pH 7.4), $100 \mathrm{mM} \mathrm{NaCl}, 1.0 \%$ Nonidet P-40, protease inhibitor cocktails (Sigma-Aldrich), and $1 \mathrm{mM}$ Phenylmethylsulfonyl fluoride, and homogenized. The cell lysates were precleared, and supernatant fluid was subjected to immunoprecipitation with an M2 anti-FLAG antibody coupled to Sepharose beads (Sigma-Aldrich) to precipitate transfected ARHGAP24. Immunoprecipitates were washed five times with the lysis buffer, and bound protein was detected by western blot analysis using anti-FLAG antibody for ARHGAP24 or anti-HA antibody for ARF6.

Statistical analysis. The statistical significance was accessed by twotailed unpaired Student's $t$-test or Welch's $t$-test (see figure legends). Differences were considered to be statistically significant at a $p<0.05$. Error bars (standard error of the mean, S.E.M) and $p$-values were determined from the results of at least three experiments.

\section{Results}

Depletion of ARHGAP24 increases the length of pseudopods induced by activated ARF6 in MDA-MB-231 cells. ARF6 controls shape change and actin remodeling of carcinoma cells through regulation of RAC activity. Several RAC guanine nucleotide exchange factors were shown to be activated downstream of ARF6 $(17,20)$, and we showed that RACGAP activity of ARHGAP24 is regulated downstream of activated ARF6 $(25,27)$. Constitutively activated ARF6 Q67L stimulates lamellae formation, which is characterized by protrusions with accumulation of actin filaments (17). We, therefore, studied whether ARHGAP24 is involved in the ARF6-mediated actin remodeling and cell shape change of human breast carcinoma MDA-MB-231 cells.

To study the role of ARHGAP24, MDA-MB-231 cells were transfected with ARHGAP24-specific siRNA. Endogenous ARHGAP24 was effectively but not completely depleted in MDA-MB-231 cells after treatment with ARHGAP24 siRNA 

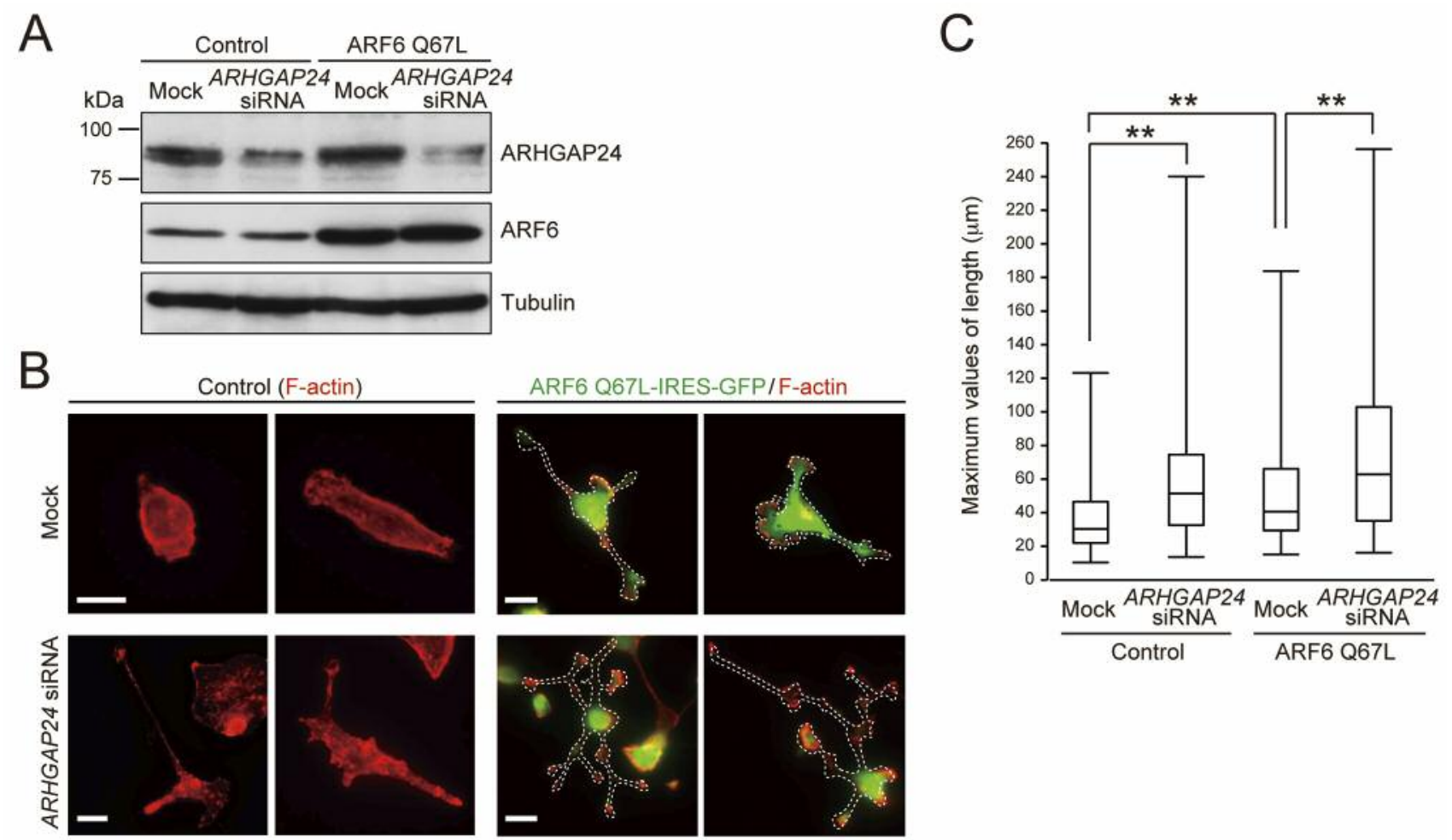

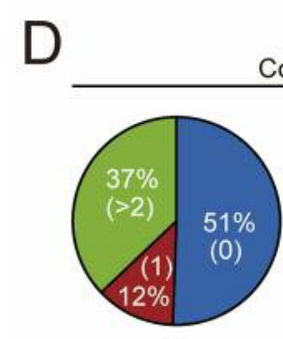

Mock
Control

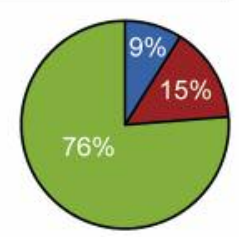

ARHGAP24 siRNA

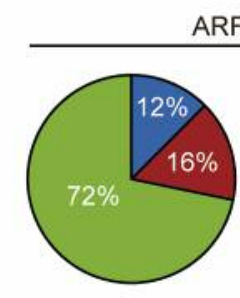

Mock
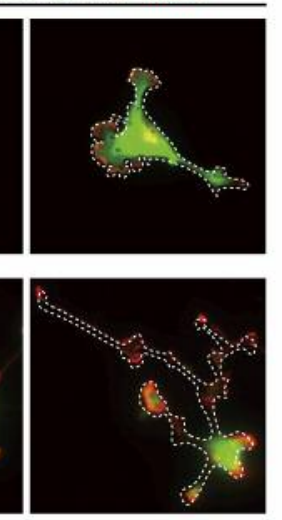

ARF6 Q67L

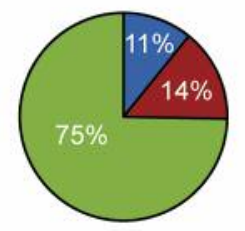

ARHGAP24 SIRNA
No pseudopod

One pseudopod

More than two pseudopods

Figure 1. Involvement of RHO GTPase activating protein 24 (ARHGAP24) in ADP ribosylation factor 6 (ARF6)-induced cell shape change. A: MDA-MB-231 cells were transfected with pIRES2-AcGFP-ARF6 Q67L in the absence or presence of ARHGAP24 siRNA. ARHGAP24 and ARF6 proteins were analyzed by immunoblotting using anti-ARHGAP24 and anti-ARF6. Tubulin was used as a loading control. B: MDA-MB-231 cells transfected as discribed in (A) on poly-L-lysine-coated coverslips were fixed and ARF6-expressing cells were identified by staining the cells with anti-green fluorescent protein (GFP) (green). F-actin was localized by Alexa Fluor 568-phalloidin (red). Representatively merged images are shown. Cell outlines are shown by white dotted lines. Scale bars, $30 \mu \mathrm{m}$. C: The maximal length of pseudopods in the cells were calculated and the data are shown as box and whisker plots ( $n=100$ cells counted for each experiment). Statistical significance was determined by Welch's $t$-test. **p<0.01. $D$ : The total number of pseudopods in the cells $(n=100)$ were calculated and the cells were categorized as having no pseudopod $(0$, blue), one pseudopod $(1$, red $)$, or more than two pseudopods ( $\geq 2$, green).

(expression less than 30\%) (Figure 1A). Non-transfected control (mock) cells exhibited flat morphology and about half the cells had polarized morphology, with one or two pseudopods (Figure 1B and D). Depletion of endogenous ARHGAP24 alone increased the number of polarized cells and the length of pseudopods (Figure 1B-D). When MDA-MB-231 cells were transfected with ARF6 Q67L alone, many cells produced lamellae and long pseudopods with actin filaments at the periphery. Depletion of ARHGAP24 in cells transfected with AFR6 Q67L significantly increased in the length of pseudopods, compared to cells transfected with ARF6 Q67L alone (Figure 1B and C). Interestingly ARHGAP24 knockdown did not increase the total number of pseudopods induced by activated ARF6 (Figure 1D). These results suggest that endogenous ARHGAP24 may have a role in blocking the extension of pseudopods induced by ARF6. 
A
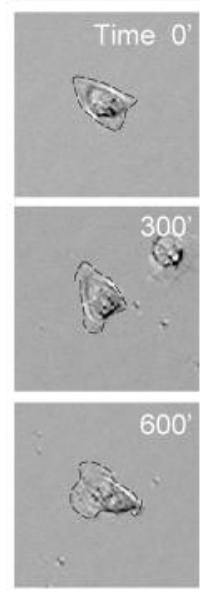

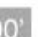
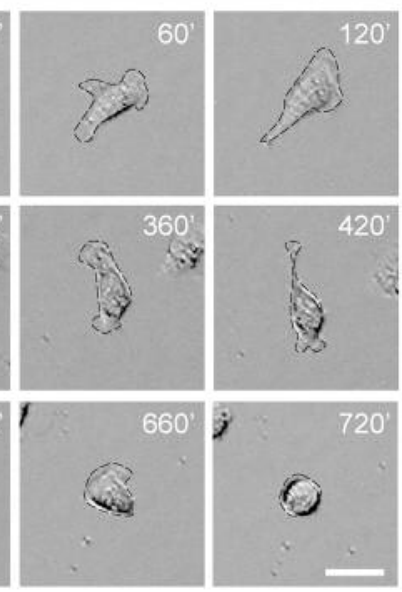

Control (Mock)
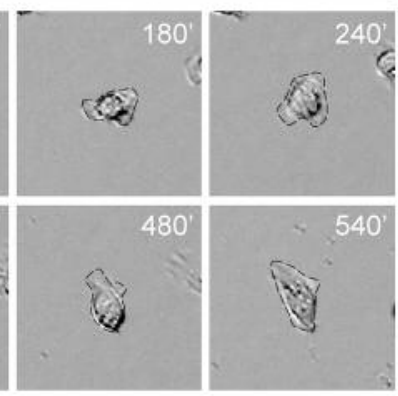

ARF6 Q67L
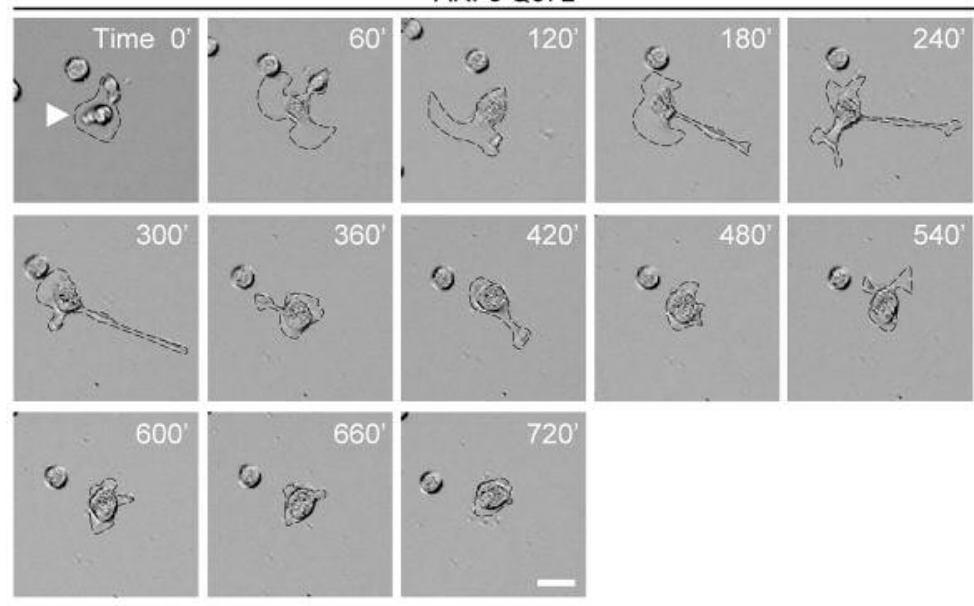

ARF6 Q67L + ARHGAP24 siRNA
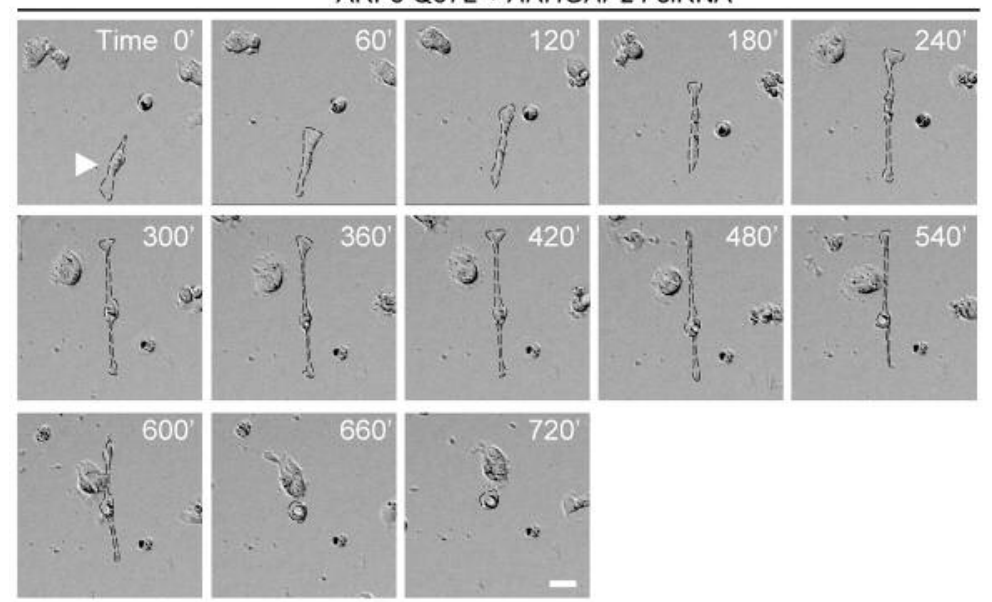

B

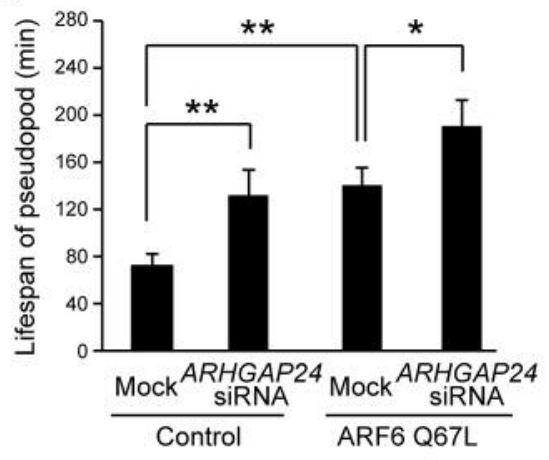

C

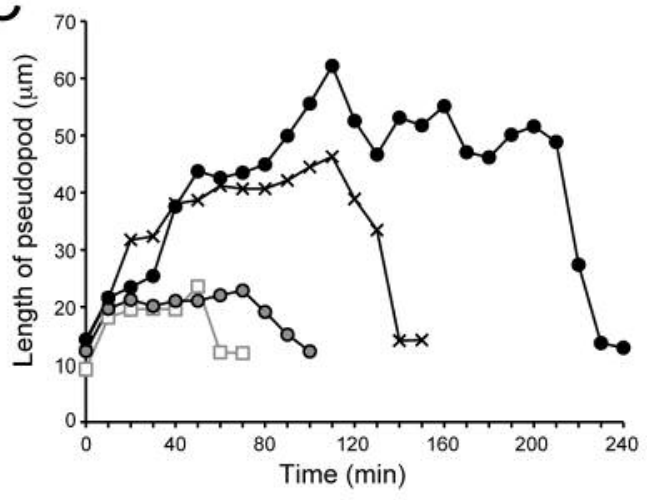

Control (mock)

O ARHGAP24 siRNA

X ARF6 Q67L (mock)

ARF6 Q67L + ARHGAP24 siRNA

Figure 2. Depletion of RHO GTPase activating protein 24 (ARHGAP24) in MDA-MB-231 cells increases the lifespan of pseudopods induced by activated ADP ribosylation factor 6 (ARF6). A: Time-lapse images of MDA-MB-231 cells expressing activated ARF6 Q67L. MDA-MB-231 cells were transfected with pIRES2-AcGFP-ARF6 Q67L in the absence or presence of ARHGAP24 siRNA on glass-bottom dishes. Images were acquired at 10min intervals and are shown from a selected 60-min interval. Arrowheads indicate the cells transfected with ARF6 Q67L. Cell outlines are shown by black dotted lines. Scale bars, $30 \mu \mathrm{m}$. B: Lifespan of individual pseudopods. The data were calculated and expressed as the mean \pm S.E.M. $(n=49-69$ pseudopods). Statistical significance was determined by Student's t-test: ${ }^{*} p<0.05$, **p<0.01. C: Length of pseudopod over time ( $n=3-5$ cells). 

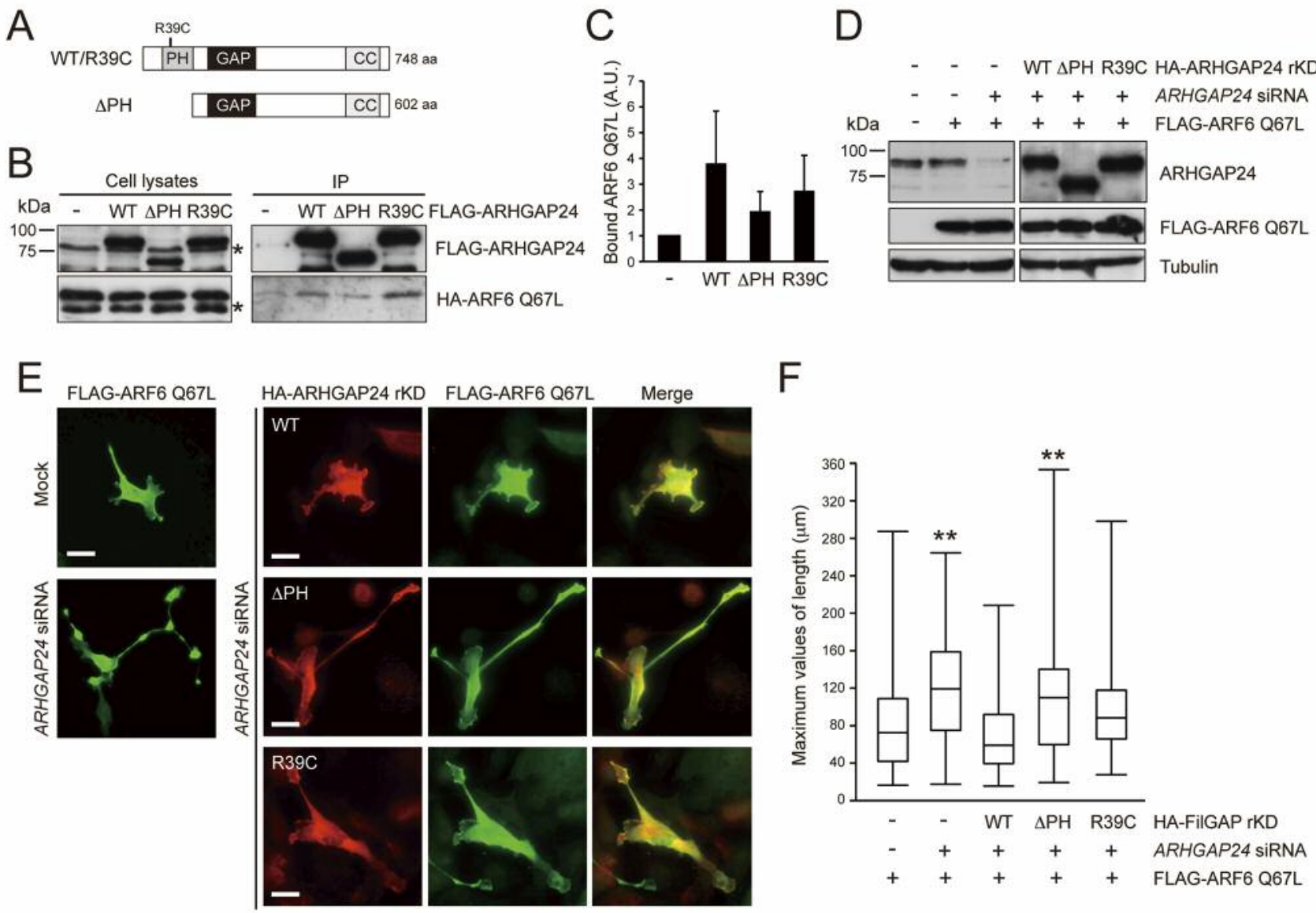

Figure 3. The pleckstrin homology $(P H)$ domain of RHO GTPase activating protein 24 (ARHGAP24) is required for retraction of pseudopods. A: Schematic diagram of mutated ARHGAP24 constructs [wild type (WT), $\triangle P H, R 39 C$ ]. B: HEK293 cells were transfected with FLAG-ARHGAP24 constructs and hepatitis A (HA)-ARF6 Q67L. Cell lysates were prepared, and FLAG-ARHGAP24 was immunoprecipitated (IP) using an anti-FLAG antibody coupled to Sepharose beads. The washed immunoprecipitates were immunoblotted for the presence of ARHGAP24 (anti-FLAG) and ARF6 Q67L (anti-HA). Asterisks indicate non-specific bands. C: The relative amount of bound ARF6 protein. The signal intensity of the bands was quantified by ImageJ. The amount of bound ARF6 (IP) was normalized to that of total ARF6 (cell lysates) and expressed as the mean $\pm S . E . M$. (N=3). A.U., Arbitrary units. D: MDA-MB-231 cells were transfected with FLAG-ARF6 Q67L or HA-ARHGAP24 constructs (WT, $\triangle P H, R 39 C)$ resistant to ARHGAP24 siRNA $(r K D)$ in the absence or presence of ARHGAP24 siRNA. FLAG-ARF6 and ARHGAP24 proteins were analyzed by immunoblotting using anti-FLAG and anti-ARHGAP24 antibodies. Tubulin was used as a loading control. E: MDA-MB-231 cells transfected as in (D) on poly-L-lysinecoated coverslips were fixed and FLAG-ARF6 and HA-ARHGAP24 proteins were localized by staining the cells with anti-FLAG (green) and anti-HA (red) antibodies. Scale bars, $30 \mu \mathrm{m}$. F: Maximal length of pseudopods in the cells were calculated and the data are shown as box and whisker plots $(n=100$ cells). Statistical significance was determined by Welch's t-test (vs. FLAG-ARF6 Q67L-expressing cells): **p<0.01.

Depletion of ARHGAP24 may stabilize and increase the lifespan of pseudopods induced by activated ARF6. In order to understand in greater detail how ARHGAP24 may affect ARF6-mediated change in cell shape, we next studied the changes in cell morphology over time using time-lapse video microscopy (Figure 2). MDA-MB-231 cells were transfected with ARF6 Q67L-IRES-GFP in the absence or presence of ARHGAP24 siRNA. Transfected cells were identified using GFP signals under microscopic observation and their live cell behavior was monitored by time-lapse phase-contrast microscopy for $12 \mathrm{~h}$. Compared to non-transfected control (mock) cells (Figure 2A), the cells transfected with constitutively activated ARF6 Q67L alone induced pseudopod formation with continuous extension and retraction (Figure 2A) and increased the average lifespan of pseudopods (Figure 2B). Depletion of ARHGAP24 in ARF6 Q67L-expressing cells produced longer pseudopods (Figure 2A) and significantly increased the average lifespan of pseudopods (Figure 2B). In order to further analyze the time course of elongation and retraction of pseudopods induced by ARF6, we monitored the behavior of cells with average pseudopod lifespan in control (mock) and ARHGAP24- 


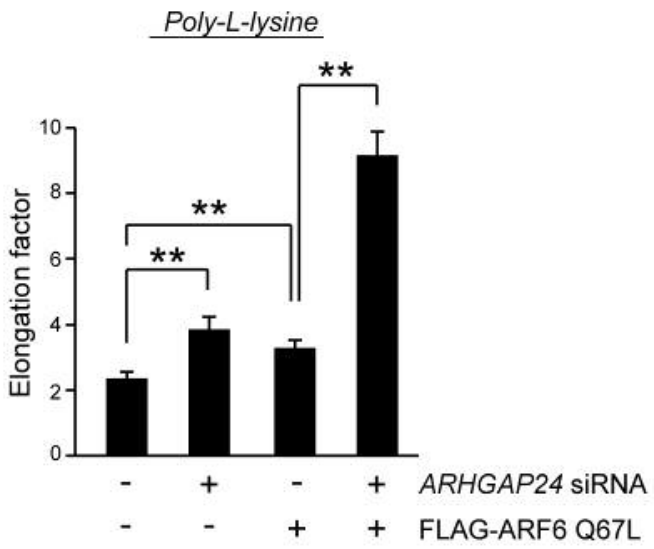

Collagen type I

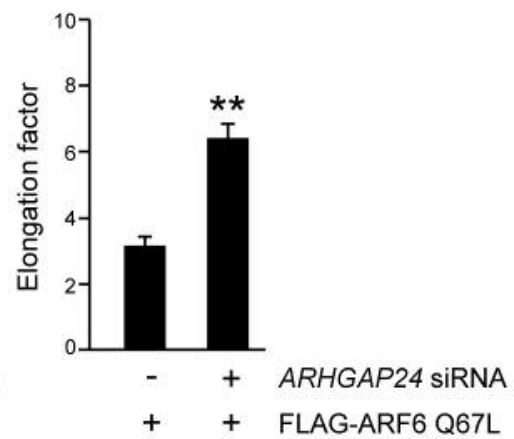

$\underline{\text { Fibronectin }}$

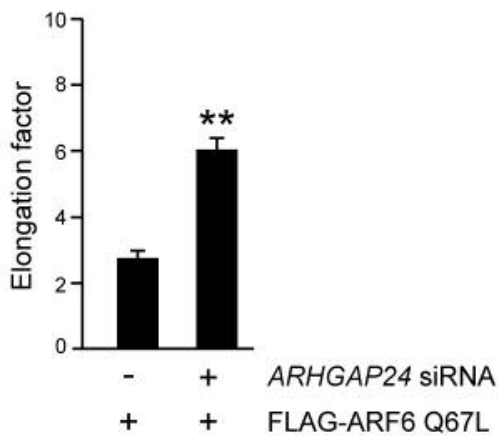

Figure 4. Depletion of RHO GTPase activating protein 24 (ARHGAP24) does not require extracellular matrix to affect ADP ribosylation factor 6 (ARF6)-mediated change in cell shape. MDA-MB-231 cells were transfected with FLAG-ARF6 Q67L in the absence or presence of ARHGAP24 siRNA on coverslips coated with poly-L-lysine, collagen type I, or fibronectin. After transfection, the cells were fixed and FLAG-ARF6 protein and $F$-actin were localized by staining the cells with anti-FLAG for ARF6 and phalloidin for F-actin. The elongation factor was calculated as the ratio of maximum length to the width of cells and the data are expressed as the mean \pm S.E.M. $(n=100$ cells). Statistical significance was determined by the Student's t-test: ${ }^{* *} p<0.01$.

depleted cells. We found that the time for which cells manage to maintain a stable pseudopod length was significantly increased in ARHGAP24-depleted cells, whereas the extension of pseudopods occurred at similar speed in control and ARHGAP24-depleted cells (Figure 2C). These results may suggest that ARHGAP24 could be involved in the retraction of pseudopods induced by ARF6.

Binding of ARHGAP24 to activated ARF6 may affect cell shape change induced by ARF6. We next examined if binding of ARHGAP24 to ARF6 is required for retraction of pseudopods induced by ARF6. We confirmed that full-length ARHGAP24 binds to activated ARF6 Q67L after immunoprecipitation from HEK293 cells transfected with cDNAs of both proteins (Figure $3 \mathrm{~A}-\mathrm{C})$. ARHGAP24 mutant lacking PH domain $(\Delta \mathrm{PH})$ did not bind to ARF6 Q67L (Figure 3A-C). Therefore the PH domain of ARHGAP24 mediates a stable complex with activated ARF6 in intact cells. ARHGAP24 R39C, a mutation resulting in deficiency in binding to phosphatidylinositol 3,4,5-trisphosphate (PIP3), was co-precipitated with constitutively activated ARF Q67L when expressed in HEK293 cells (Figure 3A-C) as reported previously (25). Thus, ARHGAP24 binds to ARF6 in a PIP3-independent manner.

We introduced five silent mutations into the siRNAtargeting sequence of ARHGAP24 (WT, $\triangle$ PH, R39C) (23) and examined whether or not formation of long pseudopods that were induced by $A R H G A P 24$ siRNA was prevented (Figure 3D). ARHGAP24 knockdown stimulated the production of long pseudopods in ARF6 Q67L-expressing cells (Figure 3E and F). Depletion of ARHGAP24 was rescued by overexpression of siRNA-resistant ARHGAP24 WT and R39C, but not $\triangle \mathrm{PH}$ (Figure $3 \mathrm{E}$ and F). This demonstrates that retraction of pseudopods induced by ARF6 may require the $\mathrm{PH}$ domain of ARHGAP24, which is the binding site of activated ARF6.

Knockdown of ARHGAP24 does not require a particular extracellular matrix (ECM) to affect ARF6-mediated change in cell shape. Finally, we examined whether the change of cell shape is dependent on particular ECM components. MDA-MB-231 cells were co-transfected with ARHGAP24 siRNA and ARF6 Q67L on collagen type I- or fibronectincoated coverslips. We quantified cell morphology by measuring the elongation factor of cells (the ratio of maximum length to width). The values for cells cultured on collagen type I and fibronectin were similar for cells cultured on poly-L-lysine-coated coverslis (Figure 4). These results suggest that ARHGAP24 may regulate change in cell shape independently from the ECM.

\section{Discussion}

In this study, we showed that ARHGAP24 might play a role in ARF6-mediated actin remodeling and change in cell shape. Overexpression of constitutively activated ARF6 Q67L in human breast carcinoma MDA-MB-231 cells produced lamellae around the cell periphery. On the other hand, depletion of endogenous ARHGAP24 in ARF6 Q67Lexpressing cells induced long pseudopods. This suggests that ARHGAP24 may control the length of pseudopods induced 
by ARF6. Previous studies have shown that ARF6 controls actin cytoskeleton through regulation of RAC (16-20). ARHGAP24 may suppress extension or stimulate retraction of pseudopods thorough down-regulation of RAC.

The time-lapse video microscopic observation revealed more details in the change of cell shape of ARHGAP24depleted cells expressing ARF6 Q67L. The extension of protrusion occurred at similar speed between control and ARHGAP24-depleted cells, whereas the time for which cells manage to maintain a stable protrusion length was significantly increased in ARHGAP24-depleted cells. These results suggest that ARHGAP24 may be involved in the retraction of pseudopods induced by ARF6.

Our study suggests that ARHGAP24 may function downstream of ARF6 to regulate the formation of pseudopods induced by ARF6. Firstly, ARHGAP24 binds to activated GTP-bound ARF6 Q67L but not GDP-bound inactive ARF6 T27N (25). Secondly, knockdown of ARHGAP24 was rescued by WT but not mutant ARHGAP24 lacking ARF6-binding site (i.e. PH domain). Moreover, the mutant ARHGAP24 R39C, which binds to ARF6 but is deficient in PIP3 binding (25), still enabled rescue from knockdown. Although the $\mathrm{PH}$ domain of ARHGAP24 may bind to factors other than PIP3 and ARF6, and could affect ARF6-dependent actin remodeling, our present study strongly suggests that binding of ARHGAP24 to ARF6 is necessary to regulate the length of pseudopods induced by ARF6.

We showed that depletion of ARHGAP24 induced elongated cell shapes in cells cultured on poly-L-lysinecoated coverslips. Similar effect of ARHGAP24 knockdown was also observed from cells cultured on collagen and fibronectin. These results suggest that signals from specific extracellular matrixes do not affect production of long pseudopods induced by knockdown of ARHGAP24 (32).

It is well established that ARF6 is involved in the regulation of cancer cell migration and invasion $(8-11,14,15)$. We showed that ARHGAP24 mediates breast cancer cell migration and invasion (23). Although ARHGAP24 appears to regulate ARF6-mediated change in cell shape, we were unable to determine the role of ARHGAP24 in ARF6-mediated cancer cell migration. MDA-MB-231 cells transfected with ARF6 Q67L induced actin-rich protrusions and showed dynamic cell shape changes. However, they did not migrate effectively on the substrates. The cells may need to maintain proper ARF6 activity in order to migrate, and expression of constitutively activated ARF6 may be inhibitory for cell migration (14). Further study is necessary to determine the role of ARHGAP24 in ARF6-mediated cancer cell migration.

In summary, we showed that ARHGAP24 may function to stabilize pseudopod formation downstream of ARF6 during ARF6-dependent change in cell shape. We present evidence that ARHGAP24 may control retraction of pseudopods through binding to activated ARF6.

\section{Conflicts of Interest}

The Authors declare no conflicts of interest.

\section{Acknowledgements}

The Authors thank K. Nakayama (Kyoto University, Japan) for HA-ARF6 constructs. This work was supported by Grants-in-Aid for Scientific Research from the Japan Society for the promotion of Science and the Ministry of Education, Culture, Sports, Science, and Technology of Japan, and Grant for All Kitasato Project Study.

\section{References}

1 Pandya P, Orgaz JL and Sanz-Moreno V: Modes of invasion during tumour dissemination. Mol Oncol 11: 5-27, 2017.

2 Sanz-Moreno V, Gadea G, Ahn J, Paterson H, Marra P, PInner S, Sahai E and Marshall CJ: Rac activation and inactivation control plasticity of tumor cell movement. Cell 135: 510-523, 2008.

3 Friedl $\mathrm{P}$ and Wolf K: Plasticity of cell migration: a multiscale tuning model. J Cell Biol 188: 11-19, 2010.

4 Burridge $\mathrm{K}$ and Wennerberg K: RHO and RAC take center stage. Cell 116: 167-179, 2004.

5 Jaffe AB and Hall A: RHO GTPases: Biochemistry and biology. Annu Rev Cell Dev Biol 21: 247-269, 2005.

6 Hall A: RHO family GTPases. Biochem Soc Trans 40: 13781382, 2012.

7 Porter AP, Papaioannou A and Malliri A: Deregulation of RHO GTPases in cancer. Small GTPases 7: 123-138, 2016.

8 Yamauchi Y, Miura Y and Kanaho Y: Machineries regulating the activity of the small GTPase ARF6 in cancer cells are potential targets for developing innovative anti-cancer drugs. Adv Biol Regul 63: 115-121, 2017.

9 Sabe H, Hashimoto S, Morishige M, Ogawa E, Hashimoto A, Nam JM, Miura K, Yano H and Onodera Y: The EGFRGEP100-ARF6-AMAP1 signaling pathway specific to breast cancer invasion and metastasis. Traffic 10: 982-993, 2009.

$10 \mathrm{Hu}$ B, Shi B, Jarzynka MJ, Yiin JJ, D'Souza-Schorey C and Cheng SY: ADP-ribosylation factor 6 regulates glioma cell invasion through the IQ-domain GTPase-activating protein 1RAC1-mediated pathway. Cancer Res 69: 794-801, 2009.

11 Muralidharan-Chari V, Hoover H, Clancy J, Schweitzer J, Suckow MA, Schroeder V, Castellino FJ, Schorey JS and D'Souza-Schorey C: ADP-ribosylation factor 6 regulates tumorigenic and invasive properties in vivo. Cancer Res 69: 2201-2209, 2009.

12 Palacios F, Schweitzer JK, Boshans RL and D'Souza-Schorey C: ARF6-GTP recruits NM23-H1 to facilitate dynamin-mediated endocytosis during adherence junctions disassembly. Nat Cell Biol 4: 929-936, 2002.

13 Powelka AM, Sun J, Li J, Gao M, Shaw LM, Sonnenberg A and Hsu VW: Stimulation-dependent recycling of integrin beta1 regulated by ARF6 and Rab11. Traffic 5: 20-36, 2004.

14 Hashimoto S, Onodera Y, Hashimoto A, Tanaka M, Hamaguchi M, Yamada A and Sabe H: Requirement for ARF6 in breast cancer invasive activities. Proc Natl Acad Sci USA 101: 66476652, 2004. 
15 Sabe H: Requirement for ARF6 in cell adhesion, migration, and cancer cell invasion. J Biochem 134: 485-489, 2003.

16 Donaldson JG and Honda A: Localization and function of ARF family GTPases. Biochem Soc Trans 33: 639-642, 2005.

17 D'Souza-Schorey C and Chavrier P: ARF proteins: roles in membrane traffic and beyond. Nat Rev Mol Cell Biol 7: 347358, 2006.

18 Myers KR and Casanova JE: Regulation of actin cytoskeleton dynamics by ARF-family GTPases. Trends Cell Biol 18: 184192, 2008.

19 Palacios F and D'Souza-Schorey C: Modulation of RAC1 and ARF6 activation during epithelial cell scattering. J Biol Chem 278: 17395-17400, 2003.

20 Santy LC, Ravichandran KS and Casanova JE: The DOCK180/ELMO complex couples ARNO-mediated ARF6 activation to the downstream activation of RAC1. Curr Biol 15: 1749-1754, 2005.

21 Ohta Y, Hartwig JH and Stossel TP: FilGAP, a RHO- and ROCK-regulated GAP for RAC binds filamin A to control actin remodelling. Nat Cell Biol 8: 803-814, 2006.

22 Ehrlicher AJ, Nakamura F, Hartwig JH, Weitz DA and Stossel TP: Mechanical strain in actin networks regulates FilGAP and integrin binding to filamin A. Nature 478: 260-263, 2011.

23 Saito K, Ozawa Y, Hibino K and Ohta Y: FilGAP, a RHO/RHOassociated protein kinase-regulated GTPase-activating protein for RAC, controls tumor cell migration. Mol Biol Cell 23: 47394750, 2012.

24 Nakamura F: FilGAP and its close relatives: a mediator of RHORAC antagonism that regulates cell morphology and migration. Biochem J 453: 17-25, 2013.

25 Kawaguchi K, Saito K, Asami H and Ohta Y: ADP ribosylation Factor 6 (ARF6) acts through FilGAP protein to down-regulate RAC protein and regulates plasma membrane blebbing. J Biol Chem 289: 9675-9682, 2014.
26 Nakahara S, Tsutsumi K, Zuinen T and Ohta Y: FilGAP, a RHOROCK-regulated GAP for RAC, controls adherens junctions in MDCK cells. J Cell Sci 128: 2047-2056, 2015.

27 Morishita Y, Tsutsumi K and Ohta Y: Phosphorylation of serine 402 regulates RACGAP protein activity of FilGAP protein. J Biol Chem 290: 26328-26338, 2015.

28 Yamada H, Tsutsumi K, Nakazawa Y, Shibagaki Y, Hattori S and Ohta Y: Src family tyrosine kinase signaling regulates FilGAP through association with RBM10. PLoS One 11: e0146593, 2016.

29 Iida T, Saito K, Katagiri K, Kinashi $\mathrm{T}$ and Ohta Y: The RACGAP protein FilGAP is a negative regulator of chemokinepromoted lymphocyte migration. FEBS Lett 590: 1395-1408, 2016.

30 Nishi T, Takahashi H, Hashimura M, Yoshida T, Ohta Y and Saegusa M: FilGAP, a RAC-specific RHO GTPase-activating protein, is a novel prognostic factor for follicular lymphoma. Cancer Med 4: 808-818, 2015.

31 Hara A, Hashimura M, Tsutsumi K, Akiya M, Inukai M, Ohta Y and Saegusa M: The role of FilGAP, a RAC-specific RHOGTPase-activating protein, in tumor progression and behavior of astrocytomas. Cancer Med 5: 3412-3425, 2016.

32 Moreno-Layseca P and Streuli CH: Signalling pathways linking integrins with cell cycle progression. Matrix Biol 34: 144-153, 2014.
Received June 19, 2017

Revised July 11, 2017

Accepted July 12, 2017 